\title{
Thermodynamics for Fractional Exclusion Statistics
}

\author{
Serguei B. Isakov ${ }^{a}$, Daniel P. Arovas ${ }^{b}$, Jan Myrheim $^{c}$, Alexios P. Polychronakos ${ }^{d}$ \\ Centre for Advanced Study, Norwegian Academy of Science and Letters, \\ Drammensveien 78, 0271 Oslo, Norway \\ and \\ ${ }^{a}$ Medical Radiological Research Center, Obninsk, Kaluga Region 249020, Russia \\ ${ }^{b}$ Physics Department 0319, University of California at San Diego, La Jolla CA 92093, USA \\ ${ }^{c}$ Department of Physics, University of Trondheim, N-7034 Trondheim, Norway \\ ${ }^{d}$ Theoretical Physics Department, Uppsala University, Box 803, S-75108 Uppsala, Sweden
}

(January 23, 1996)

\begin{abstract}
We discuss the thermodynamics of a gas of free particles obeying Haldane's exclusion statistics, deriving low temperature and low density expansions. For gases with a constant density of states, we derive an exact equation of state and find that temperature-dependent quantities are independent of the statistics parameter.
\end{abstract}

PACS numbers: 05.30.-d, 05.70.Ce

Keywords: Exclusion Statistics, Thermodynamics, Equation of State 
Haldane's definition of fractional statistics [1] is based on the generalized exclusion principle $\Delta d(N)=-g \Delta N$, where $d(N)$ is the dimension of the single particle Hilbert space when $N-1$ states are occupied and $g$ is the exclusion statistics parameter $\left(g_{\text {Bose }}=0, g_{\text {Fermi }}=1\right)$. This leads to a statistical weight

$$
W=\frac{[K-(g-1)(N-1)] !}{N ![K-g(N-1)-1] !} ; \quad K=d(1)
$$

which, when applied locally in phase space (i.e. to a set of neighboring energy levels), results in distribution functions for free particles obeying fractional exclusion statistics (FES) [2 [4. Corresponding thermodynamic properties have been discussed [3,5]. Remarkably, the single-state grand partition functions for FES previously appeared as describing the thermodynamics of anyons in the lowest Landau level [6], and connection with FES was then established [3]. The FES distributions were also derived [7] for the elementary excitations of the Calogero [11] and Sutherland [12] models; the latter models may be viewed as systems with purely "statistical" interactions [13, 14]. FES is sometimes called ideal FES to distinguish it from exclusion statistics with nonlocal statistical interactions related to more general integrable models [8,15]. Connection with integrable models was used to derive thermodynamic quantities for exclusion statistics in one dimension [16]. Note also that the idea of generalized exclusion principle has been recently extended resulting in statistical weights different from (11) [17 19].

The original suggestion that fractional quantum Hall effect quasiparticles obey FES [1] has since been verified numerically [20,21] and analytically [22]. There were also speculations as to the applicability of FES to other models of condensed matter systems [5.23,24, in which context the Thomas-Fermi method was adapted to study FES [25].

In this paper we discuss the thermodynamics of a gas of free (spinless) particles obeying FES, deriving Sommerfeld and virial expansions, extending previous work by Nayak and Wilczek [5] on this subject. In particular, we derive several new analytic results. For gases with a constant density of levels, we obtain an explicit expression for the energy density as a function of particle density and temperature. We find in this case that, at low temperatures, 
the heat capacity is exactly linear (up to nonanalytic corrections) and independent of the statistics parameter $g$. In the low density expansion, only the second virial coefficient is $g$-dependent.

General Relations — The occupation distribution function derived from eq. (1) is

$$
n(\varepsilon, \mu, T ; g)=\frac{w}{1+(g-1) w}
$$

where $w$ satisfies

$$
g \ln (1-w)-\ln w=\frac{\varepsilon-\mu}{k_{\mathrm{B}} T}
$$

The grand potential is then a sum over the single-particle states $i$ :

$$
\Omega(T, V, \mu)=k_{\mathrm{B}} T \sum_{i} \ln \left(1-w_{i}\right)
$$

The distribution function $n_{i}$ and the single state grand partition function $\xi_{i}$, related by $n_{i}=x_{i}\left(\partial / \partial x_{i}\right) \ln \xi_{i}$, with $x_{i} \equiv \exp \left[\left(\mu-\varepsilon_{i}\right) / k_{\mathrm{B}} T\right]$, can be expanded in powers of $x_{i}$,

$$
\xi_{i}=\frac{1}{1-w_{i}}=\sum_{l=0}^{\infty} P_{l} x_{i}^{l}, \quad n_{i}=\sum_{l=1}^{\infty} Q_{l} x_{i}^{l},
$$

where [7]

$$
P_{l}=\prod_{k=2}^{l}\left(1-g \frac{l}{k}\right), \quad Q_{l}=\prod_{k=1}^{l-1}\left(1-g \frac{l}{k}\right) .
$$

These coefficients, which may be introduced as a priori probabilities [17], generally become negative for certain values of $g$. One can implement a microscopic formulation of FES which does not rely on negative probabilities [26].

Consider a gas of free FES particles in $D$ dimensions with dispersion $\varepsilon(p)=a p^{\sigma}$. The pressure is given by

$$
P=-\Omega / V=\Delta \int_{0}^{\infty} d \varepsilon \varepsilon^{D / \sigma} n(\varepsilon)
$$

where $\Delta=a^{-D / \sigma} /(2 \sqrt{\pi} \hbar)^{D} \Gamma\left(1+\frac{1}{2} D\right)$. Note that $P=(\sigma / D) E / V$, where $E=\sum_{i} \varepsilon_{i} n_{i}$ is the energy. It is convenient to express integrals of the form 


$$
I[f]=\int_{0}^{\infty} d \varepsilon f(\varepsilon) n(\varepsilon)
$$

as ones over the variable $w$,

$$
I[f]=k_{\mathrm{B}} T \int_{0}^{w(0)} \frac{d w}{1-w} f\left(\mu+g k_{\mathrm{B}} T \ln (1-w)-k_{\mathrm{B}} T \ln w\right),
$$

where $w(0)$ satisfies Eq. (3) with $\varepsilon=0$.

Low Temperatures - At low temperatures, Eq. (9) results in the Sommerfeld expansion

$$
\begin{aligned}
I[f] & =g^{-1} \int_{k_{\mathrm{B}} T \ln w(0)}^{\mu} d \varepsilon f(\varepsilon)+\sum_{l=0}^{\infty} \frac{\left(k_{\mathrm{B}} T\right)^{l+1}}{l !} C_{l}(g) f^{(l)}(\mu), \\
C_{l}(g) & =\int_{0}^{w(0)} \frac{d w}{1-w}\left\{[g \ln (1-w)-\ln w]^{l}-[g \ln (1-w)]^{l}\right\} .
\end{aligned}
$$

Here $w(0)=1-\mathcal{O}\left(e^{-\mu / g k_{\mathrm{B}} T}\right)$ and can be set to unity, up to nonperturbative corrections (it is necessary that $g>0$ ). Our result for $C_{l}(g)$ is equivalent to that derived in Ref. [5], but in its present form we clearly see that $C_{l}(g)$ is a polynomial of order $(l-1)$ in $\mathrm{g}$,

$$
\begin{gathered}
C_{l}(g)=\sum_{k=0}^{l-1} C_{l, k} g^{k} \\
C_{l, k}=(-1)^{l-k}\left(\begin{array}{l}
l \\
k
\end{array}\right) \int_{0}^{1} \frac{d w}{w} \ln ^{k}(w) \ln ^{l-k}(1-w) .
\end{gathered}
$$

It is now straightforward to derive the duality relation

$$
C_{l, k}=(-1)^{l-1} C_{l, l-k-1}
$$

as well as the results

$$
\begin{gathered}
C_{0}(g)=0, \quad C_{1}(g)=\frac{\pi^{2}}{6}, \quad C_{2}(g)=2 \zeta(3)(1-g), \\
C_{3}(g)=\frac{\pi^{4}}{15}\left(1-\frac{1}{4} g+g^{2}\right) .
\end{gathered}
$$

This proves the conjecture $C_{0}(g)=0$ (see also Ref. [17]) and agrees with the numerical estimates of $C_{1}\left(\frac{1}{2}\right)$ and $C_{2}\left(\frac{1}{2}\right)$ from Ref. [5]

From the Sommerfeld expansions for the particle number and the energy, we find a chemical potential 


$$
\mu=\mu_{0}\left\{1-\frac{\pi^{2}}{6} g\left(\frac{D}{\sigma}-1\right)\left(\frac{k_{\mathrm{B}} T}{\mu_{0}}\right)^{2}+\ldots\right\}
$$

and a specific heat

$$
\frac{C_{V, N}}{V}=\frac{g D n}{\sigma}\left(\frac{k_{\mathrm{B}} T}{\mu_{0}}\right)\left\{\frac{\pi^{2}}{3}+6 \zeta(3)(1-g)\left(\frac{D}{\sigma}-1\right)\left(\frac{k_{\mathrm{B}} T}{\mu_{0}}\right)+\ldots\right\},
$$

where $\mu_{0}=(g \rho / \Delta)^{\sigma / D}$ is the $T=0$ chemical potential and $\rho=N / V$ is the particle density.

Examples - When $\varepsilon(p)=p^{2} / 2 m$, one obtains a compressibility $\kappa=D / 2 n \mu_{0}$ and a sound velocity $v_{s}=\sqrt{2 \mu_{0} / m D}$. Restricting further to the case $D=1$, the sound velocity $v_{s}=\pi \hbar g n / m$ corresponds to the pseudo Fermi velocity $v_{F}=p_{F} / m\left(n(p)=1 / g\right.$ for $|p| \leq p_{F}$, otherwise $n(p)=0$ for $T=0)$. The specific heat is

$$
\frac{C_{V, N}}{V}=\frac{\pi k_{\mathrm{B}}^{2} T}{3 \hbar v_{s}}-\frac{6 \zeta(3)}{\pi}(1-g) \frac{k_{\mathrm{B}}^{3} T^{2}}{m \hbar v_{s}^{3}}+\ldots
$$

This calculation provides a way to evaluate thermodynamic quantities (without restriction only to leading terms) for the Sutherland model (nonrelativistic particles on a ring of length $L$ interacting via $V\left(x_{i}-x_{j}\right)=\lambda(\lambda-1)\left(\pi^{2} / m L^{2}\right) / \sin ^{2}\left[\pi\left(x_{i}-x_{j}\right) / L\right]$ [12]). Indeed, one may interpret the thermodynamic Bethe Ansatz equations for the family of solutions of the Sutherland model with $|\Psi| \propto\left|x_{i}-x_{j}\right|^{\lambda}$ as $\left|x_{i}-x_{j}\right| \rightarrow 0(\lambda \geq 0)$ as describing an ideal gas of nonrelativistic pseudoparticles obeying $D=1$ FES with $g=\lambda$, with corresponding thermodynamic properties [7 9]. The leading term of the low temperature heat capacity known for the Sutherland model [12] is recovered by the first term in (14) (which itself, along with the above sound velocity $v_{s}$ and compressibility $\kappa=1 / n m v_{s}^{2}$, manifests in thermodynamics a bosonization of the Sutherland model [27,28]).

Cluster and Virial Expansions - For the general dispersion $\varepsilon(p)=a p^{\sigma}$, expanding Eq. (田) in powers of $x_{i}$ and making summation over $i$, we derive a cluster expansion

$$
-\frac{\Omega}{k_{\mathrm{B}} T}=\sum_{l=1}^{\infty} b_{l} z^{l}, \quad b_{l}=\frac{Q_{l}}{l} Z_{1}(T / l)
$$

where $z=e^{\mu / k_{\mathrm{B}} T}$ is the fugacity, and $Z_{1}(T)=V \Delta \Gamma(1+D / \sigma)\left(k_{\mathrm{B}} T\right)^{D / \sigma}$ is the one-particle partition function $\sum_{i} e^{-\varepsilon_{i} / k_{\mathrm{B}} T}$. One then derives in the usual way the virial expansion 


$$
\begin{aligned}
\frac{P}{k_{\mathrm{B}} T} & =\sum_{l=1}^{\infty} a_{l}(T) \rho^{l}, \quad a_{l} \equiv \tilde{a}_{l} V^{l-1}, \\
\tilde{a}_{1} & =1, \quad \tilde{a}_{2}=-\tilde{b}_{2}, \quad \tilde{a}_{3}=-2 \tilde{b}_{3}+4 \tilde{b}_{2}^{2} \\
\tilde{a}_{4} & =-3 \tilde{b}_{4}+18 \tilde{b}_{3} \tilde{b}_{2}-20 \tilde{b}_{2}^{3}, \quad \text { etc. }
\end{aligned}
$$

and $\tilde{b}_{l} \equiv b_{l} / b_{1}^{l}$. For example, we find

$$
a_{2}=2^{-D / \sigma}\left(g-\frac{1}{2}\right) \frac{V}{Z_{1}(T)}
$$

with linear dependence on the statistics parameter, and

$$
a_{3}=\left\{\left(4^{-D / \sigma}-\frac{2}{3} \cdot 3^{-D / \sigma}\right)+g(g-1)\left(4^{1-D / \sigma}-3^{1-D / \sigma}\right)\right\}\left(\frac{V}{Z_{1}(T)}\right)^{2} .
$$

For nonrelativistic particles and $D=1$, these expressions with $g=\lambda$ recover the virial coefficients for the Sutherland model $[29 \|$.

Gas with Constant Density of States - In the cases $D=\sigma$, the density of states is constant in energy and one can evaluate the equation of state exactly. The integral (9) applied for the particle number yields $w(0)=1-e^{-\rho / \Delta k_{\mathrm{B}} T}$. From Eq. (3), we then find

$$
\mu(\rho, T)=\frac{g \rho}{\Delta}+k_{\mathrm{B}} T \ln \left(1-e^{-\rho / \Delta k_{\mathrm{B}} T}\right) .
$$

Finally, applying the integral (9) for the pressure, we obtain

$$
P(\rho, T)=\frac{g \rho^{2}}{2 \Delta}+\frac{1}{\Delta} \int_{0}^{\rho} d \rho^{\prime} \frac{\rho^{\prime}}{\exp \left(\rho^{\prime} / \Delta k_{\mathrm{B}} T\right)-1}
$$

This result can be expanded to give low temperature and low density series. Remarkably, for $\rho \gg \Delta k_{\mathrm{B}} T$, the full perturbative result is

$$
P=\frac{E}{V}=\frac{g \rho^{2}}{2 \Delta}+\frac{\pi^{2}}{6} \Delta\left(k_{\mathrm{B}} T\right)^{2}
$$

and hence $C_{V, N} / V=\frac{1}{3} \pi^{2} \Delta k_{\mathrm{B}}^{2} T$ to all orders in $T$. In the low density limit, we find

$$
\frac{P}{k_{\mathrm{B}} T}=\rho+\frac{1}{4}(2 g-1) \frac{\rho^{2}}{\Delta k_{\mathrm{B}} T}+\sum_{l=2}^{\infty} \frac{\mathcal{B}_{l}}{(l+1) !} \frac{\rho^{l+1}}{\left(\Delta k_{\mathrm{B}} T\right)^{l}},
$$

where $\mathcal{B}_{l}$ is the $l^{\text {th }}$ Bernoulli number $\left(\mathcal{B}_{2}=\frac{1}{6}, \mathcal{B}_{4}=-\frac{1}{30}\right.$, etc. $)$, vanishing for $k$ odd. 
As is seen from Eq. (21), only the second virial coefficient is $g$-dependent. A similar claim about a virial expansion was made in Ref. [10] for the Calogero model (particles with an inverse square interaction in a harmonic potential $\frac{1}{2} m \omega^{2} x^{2}$ on a line) [11], which is a realization of FES and has a constant density of states in the quasicontinuous limit $\hbar \omega \ll k_{\mathrm{B}} T$. We should note however that the equation of state for the Calogero model, because of the presence of a harmonic potential, cannot be directly related to the above calculations for a free gas (see also discussion in Refs. [29,30]). We also notice a formal analogy between the above thermodynamic properties of free gases with $D=\sigma$ and those obtained by Sen and Bhaduri for $D=1 \mathrm{FES}$ particles in a harmonic potential in the Thomas-Fermi approximation [25], where the density of states is also constant.

The above results imply that for $D=\sigma$ only $T=0$ quantities depend on the statistics parameter. The case $D=\sigma$, in particular, corresponds to a gas of nonrelativistic particles in two dimensions, in which case $\Delta k_{\mathrm{B}} T$ is equal to $\lambda_{T}^{-2}$, where $\lambda_{T}=\hbar \sqrt{2 \pi / m k_{\mathrm{B}} T}$ is the thermal de Broglie wavelength (for the latter case, the virial expansion (21) was conjectured in Ref. [7]). It follows that investigations of whether FES occurs in two-dimensional systems (see e.g. Ref. [5]) should refer to $T=0$ properties.

In conclusion, we have derived explicitly a few first terms of both low temperature and low density expansions for gases of free spinless particles obeying FES. For gases with a constant density of states in energy, we derived the exact equation of state (19), which implies that the finite-temperature properties are identical to those for a Fermi gas and the statistics only appears in the zero-temperature quantities.

S.B.I. is grateful to J.M. Leinaas, S. Mashkevich, and S. Ouvry for stimulating discussions. We would like to thank the Centre for Advanced Study (Oslo) for kind hospitality and financial support. 


\section{REFERENCES}

[1] F.D.M. Haldane, Phys. Rev. Lett. 67 (1991) 937.

[2] S.B. Isakov, Mod. Phys. Lett. B 8 (1994) 319.

[3] Y.-S. Wu, Phys. Rev. Lett. 73 (1994) 922.

[4] A.K. Rajakopal, Phys. Rev. Lett. 74 (1995) 1048.

[5] C. Nayak and F. Wilczek, Phys. Rev. Lett. 73 (1994) 2740.

[6] A. Dasnières de Veigy and S. Ouvry, Phys. Rev. Lett. 72 (1994) 600.

[7] S.B. Isakov, Int. J. Mod. Phys. A 9 (1994) 2563.

[8] D. Bernard and Y.-S. Wu, in: New developments of integrable systems and long-ranged interaction models, eds. M.L. Ge and Y.-S. Wu (World Scientific, Singapore, 1995).

[9] Z.N.C. Ha, Phys. Rev. Lett. 73 (1994) 1574; Nucl. Phys. B 435 (1995) 604.

[10] M.V.N. Murthy and R. Shankar, Phys. Rev. Lett. 73 (1994) 3331.

[11] F. Calogero, J. Math. Phys. 10 (1969) 2191; 10 (1969) 2197; 12 (1971) 419.

[12] B. Sutherland, J. Math. Phys. 12 (1971) 246; 12 (1971) 251; Phys. Rev. A 4 (1971) 2019; 5 (1972) 1372.

[13] J.M. Leinaas and J. Myrheim, Phys. Rev. B 37 (1988) 9286; Int. J. Mod. Phys. B 5 (1991) 2573.

[14] A. Polychronakos, Nucl. Phys. B 324 (1989) 597.

[15] S.B. Isakov, Phys. Rev. Lett. 73 (1994) 2150.

[16] T. Fukui and N. Kawakami, Phys. Rev. B 51 (1995) 5239; J. Phys. A 28 (1995) 6027.

[17] A.P. Polychronakos, Phys. Lett. B 365 (1996) 202.

[18] W. Chen, Y.J. Ng, and H. van Dam, hep-th/9503236. 
[19] A.V. Ilinskaia, K.N. Ilinski, and J.M.F. Gunn, Nucl. Phys. B 458 (1996) 562.

[20] M.D. Johnson and G.S. Canright, Phys. Rev. B 49 (1994) 2947.

[21] S. He, X.C. Xie, and F.C. Zhang, Phys. Rev. Lett. 68 (1992) 3460.

[22] D. Li and S. Ouvry, Nucl. Phys. B 430 (1994) 563.

[23] R.K. Bhaduri, R.S. Bhalerao, and M.V.N. Murthy, cond-mat/9411112.

[24] R.K. Bhaduri, M.V.N. Murthy, and M.K. Srivastava, Phys. Rev. Lett. 76 (1996) 165.

[25] D. Sen and R.K. Bhaduri, Phys. Rev. Lett. 74 (1995) 3912.

[26] S.B. Isakov, Phys. Rev. B 53 (1996) 6585.

[27] F.D.M. Haldane, Phys. Lett. A 81 (1991) 153.

[28] N. Kawakami and S.-K. Yang, Phys. Rev. Lett. 67 (1991) 2493.

[29] A. Dasnières de Veigy and S. Ouvry, Phys. Rev. Lett. 75 (1995) 352.

[30] M.V.N. Murthy and R. Shankar, Phys. Rev. Lett. 75 (1995) 353. 